\title{
Recapitulating cranial osteogenesis with neural crest cells in 3-D microenvironments
}

Bumjin Namkoong ${ }^{1,2^{*}}$, Sinan Guven ${ }^{3,4^{*}}$, Shwathy Ramesan ${ }^{5}$, Volha Liaudanskaya ${ }^{3}$, Arhat Abzhanov $^{1,6 \#}$ and Utkan Demirci ${ }^{3,5 \#}$

${ }^{1}$ Department of Organismic and Evolutionary Biology, Harvard University, Cambridge, MA 02138, USA

2 Department of Molecular and Cellular Biology, Harvard University, Cambridge, MA 02138, USA

3 Demirci BAMM Labs, Canary Center at Stanford for Early Cancer Detection, Department of Radiology, Department of Electrical Engineering (By courtesy), Stanford School of Medicine, Palo Alto, CA 94304, USA

4 Izmir Biomedicine and Genome Center, Dokuz Eylul University Health Campus, Balcova, 35350, Izmir, Turkey

5 Demirci BAMM Labs, Department of Medicine, Brigham and Women's Hospital, Harvard Medical School, Boston, MA 02115, USA

6 Department of Life Sciences, Imperial College London, Silwood Park Campus Buckhurst Road, Ascot, Berkshire SL5 7PY, United Kingdom

* These authors contributed equally to this work \# Corresponding authors contributed equally UD: utkan@stanford.edu AA: abzhanov@fas.harvard.edu

Keywords: 3-D Culture, Controlling 3-D tissue microenvironment, Ectodermal to mesodermal differentiation, Bone 


\section{Abstract}

The experimental systems that recapitulate the complexity of native tissues and enable precise control over the microenvironment are becoming essential for the pre-clinical tests of therapeutics and tissue engineering. Here, we described a strategy to develop an in vitro platform to study the developmental biology of craniofacial osteogenesis. In this study, we directly osteo-differentiated cranial neural crest cells (CNCCs) in a 3-D in vitro bioengineered microenvironment. Cells were encapsulated in the gelatin-based photo-crosslinkable hydrogel and cultured up to three weeks. We demonstrated that this platform allows efficient differentiation of p75 positive CNCCs to cells expressing osteogenic markers corresponding to the sequential developmental phases of intramembranous ossification. During the course of culture, we observed a decrease in the expression of early osteogenic marker Runx2, while the other mature osteoblast and osteocyte markers such as Osterix, Osteocalcin, Osteopontin and Bone sialoprotein increased. We analyzed the ossification of the secreted matrix with alkaline phosphatase and quantified the newly secreted hydroxyapatite. The Field Emission Scanning Electron Microscope (FESEM) images of the bioengineered hydrogel constructs revealed the native-like osteocytes, mature osteoblasts, and cranial bone tissue morphologies with canaliculus-like intercellular connections. This platform provides a broadly applicable model system to potentially study diseases involving primarily embryonic craniofacial bone disorders, where direct diagnosis and adequate animal disease models are limited. 


\section{Introduction}

Tissues and organs comprise cells and intercellular components that are highly organized at the organismic level, which have a dynamic, three-dimensional (3-D) nature especially highlighted during embryonic development. Tissue functionality arises from the finely orchestrated interactions of all such elements, which are regulated by the multitude of chemical and physical stimuli [1-4]. Tissue engineering and regenerative medicine hold a great promise to create models that mimic the natural biological tissue microenvironments and recapitulate tissue and organ formation and maturation. Cellencapsulating 3-D tissue models are emerging tools that bring inspiring in vitro venues to study the development of complex physiological systems. These models overcome the limitations of the two-dimensional (2-D) monolayer cultures in studying the significantly complex biological mechanisms involved in multicellular behavior $[5,6]$. Microscale tissue models can efficiently utilize rare cells that are challenging for monolayer expansion [7]. Such microscale models are capable of recapitulating regulatory dynamics of the germ layers and cellular condensations forming at the early developmental stages. The design and fabrication of 3-D microenvironments with welldefined architecture and material compositions specific for progenitor cell types are essential for mimicking intricate events taking place in vivo. Cell-encapsulating hydrogel microenvironments are fabricated by gelation of extracellular matrix mimicry polymer solutions using various techniques including thermal, enzymatic, chemical and UV photo-crosslinking methods. In particular, the flexibility to fine-tune the microscale tissue size and shape, together with the high throughput and reproducibility aspect of the UV photo-crosslinking platform, underscores its broad utility to create successful tissue models $[8,9]$.

Often referred to as the "fourth embryonic germ layer" $[10,11]$, neural crest is a transient, multipotent embryonic population of cells, which migrate from their site of 
origin at the dorsal neural tube and differentiate into a diverse array of specialized cell types during vertebrate development [12]. Cranial neural crest cells (CNCCs) can differentiate into chondrogenic and osteogenic cells that, in turn, generate most of the cranial skeletal tissues (both bones and cartilages) in the vertebrate head $[12,13]$. Cranial dermal bones are unique in the way they are formed - dermal bones form directly from the neural crest of the cranial dermis without forming a cartilage precursor, through a process known as intramembranous ossification [12, 14-20]. Despite these unique developmental features of the cranial bones, most of our current understanding of bone development, in general, is from studies on the limb and trunk bones of mesodermal origin. The skeletogenesis using CNCCs, and the morphogenesis of cranial bone formation, are not very well understood, particularly at the tissue level, at least in part due to the lack of 3-D microscale tools to model and manipulate cranial skeletal tissues in vitro.

Recent studies investigating cranial neural crest cell biology utilize 2-D monolayer culture models, which do not replicate the native, three-dimensionally defined microenvironments [21-23]. In this study, we created a broadly applicable platform that allows the direct differentiation of multipotent progenitor cells, including those of neural crest origin. We produced in vitro 3-D tissue model constructs with relatively high throughput efficiency to mimic the osteogenic differentiation conditions of the mammalian post-migratory CNCCs (Figure 1). The objective was to recapitulate the in vivo development of cranial dermal bone tissue from CNCCs in well-defined 3-D biomimicking hydrogel units. Such a platform provides in vitro study models for embryonic craniofacial disorders, where our understanding of etiology is limited, and few adequate animal disease models exist. To this purpose, we have developed an array of microenvironments allowing successful 3-D direct differentiation of neural crest cells, forming cranial skeletal tissues and mimicking the embryonic phases of organogenesis. 


\section{Materials and Methods}

\subsection{Mouse Cranial Neural Crest Cell Isolation and Culture}

Mouse cranial neural crest cells were isolated accourding to Etchevers H. 2011 [24] under Harvard IACUC, Animal Experimentation Protocol No 26-04. Briefly, frontonasal, maxillary and mandibular prominences of E10.5 mouse embryos were dissected into small pieces in a petri dish containing DMEM/F12 media (Life Technologies) with $1 \%$ penicilin-streptomycin (Life Technologies). These tissue blocks were digested for 5 min in a mixture of warm $0.1 \%$ collagenase (Sigma) and $0.025 \%$ trypsin (Thermo Scientific), while being pipetted gently up and down to generate single cell suspension of mouse CNCCs. These cells were washed with fresh medium to stop the digestion, counted and seeded in laminin-coated plates. Cells were maintained and expanded in a monolayer culture in DMEM/F12 media with N2 supplement (Life Technologies), $10 \mathrm{ng} / \mathrm{ml}$ EGF and $10 \mathrm{ng} / \mathrm{ml}$ bFGF recombinant proteins (R\&D Systems).

\subsection{Synthesis of methacrylated gelatin hydrogel}

Gelatin (Sigma) was dissolved in $50{ }^{\circ} \mathrm{C}$ phosphate buffered saline (PBS) with $10 \%(\mathrm{w} / \mathrm{v})$ of final concentration. Methacrylic anhydride (Sigma) was added $(0.8 \mathrm{ml} / \mathrm{g})$ drop wise to the dissolved gelatin and stirred for two and a half hours. The unreacted methacrylic anhydride was dialyzed, and the final product was lyophilized. Methacrylation was monitored and controlled with NMR analysis (Oxford Instruments, NMR AS600) in deuterium oxide (Supplementary Figure 1).

\subsection{Cell encapsulation}

Cell-encapsulated 3-D hydrogels were generated using methacrylated gelatin (GelMA) as a prepolymer solution. CNCCs between passage 3 and 5 were resuspended in $5 \%$ 
(w/v) GelMA prepolymer solution containing $0.3 \% \quad(w / v)$ 2-hydroxy-1-(4(hydroxyethoxy)phenyl)-2-methyl-1- propanone photoinitiator (Irgacure 2959; CIBA Chemicals) with the cell densities of $1 \times 10^{6}, 5 \times 10^{6}, 1 \times 10^{7}$ or $4 \times 10^{7}$ cells $/ \mathrm{ml}$. The mixture of the cell-prepolymer solution was placed between $150 \mu \mathrm{m}$-thick spacers and covered with a glass coverslip (Figure 1). The geometry of the hydrogel units was shaped by a photomask with circular motifs of $500 \mu \mathrm{m}$ in diameter, placed on top of the glass coverslip. The prepolymer solution with cells was crosslinked with UV light $\left(1 \mathrm{~mW} / \mathrm{cm}^{2}\right)$ for 20 seconds, and the excess uncrosslinked polymer solution was washed out with PBS. The resulting cell-encapsulated 3-D hydrogels were cultured in osteogenic media (OM) composed of alpha-MEM (Life Technologies), 10\% fetal bovine serum (Life Technologies), $100 \mathrm{U} / \mathrm{mL}$ penicillin (Life Technologies), $100 \mathrm{mg} / \mathrm{mL}$ streptomycin (Life Technologies), $100 \mathrm{nM}$ dexamethasone (Sigma), $10 \mathrm{mM} \beta$-glycerophosphate (Sigma), $200 \mu \mathrm{M}$ ascorbic acid (Sigma) and $50 \mathrm{ng} / \mathrm{ml}$ BMP2 (R\&D Systems) for direct differentiation toward osteogenic lineage. These cell-encapsulated 3-D hydrogels were harvested weekly for three weeks for further analysis. 3-D hydrogel samples cultured in the neural crest growth media (control media, CM) were used as controls.

\subsection{Characterization of cranial neural crest cells}

Prior to 3-D hydrogel encapsulation, the isolated and monolayer-expanded postmigratory CNCCs were characterized with ectoderm- and mesenchyme-specific cell surface markers. Cells were stained with p75 antibody conjugated with fluorescein isothiocyanate (FITC) (Abcam) and CD73 antibody conjugated with phycoerythrin (PE) (BD Biosciences) in 1\% BSA solution for 40 min at $4{ }^{\circ} \mathrm{C}$ and analyzed using flow cytometry (BD FACS Calibur) and FlowJo software (Figure 2A). Cells stained with IgG FITC and IgG PE were used as negative controls. The viability and proliferation of the 
encapsulated CNCCs in 3-D in vitro culture were assessed with Live-Dead staining (Life Technologies).

\subsection{Quantitative Real-Time PCR}

Total RNA (3 replicates for each group) was extracted by TRIzol ${ }^{\circledR}$ (Life Technologies) and RNeasy MINI kit with QIAshedder columns (Qiagen) according to the manufacturer's instructions. After extraction, total RNA was resuspended in the final volume of $30 \mu \mathrm{l}$ with RNAse-free water and quantified using spectrophotometer (Nanodrop ND-1000, Delaware, USA). For the best results in the PCR array, all RNA samples were selected for purity using an A260/A280 UV spectrophotometry ratio greater the 1.99. $1 \mu \mathrm{g}$ of total RNA was used for the reverse transcription reaction using High-Capacity cDNA Reverse Transcription Kit (Applied Biosystems). Amplification and detection were carried out using SYBR Green (Kapa Biosystems) with Eppendorf Mastercycler using 2-step cycle for 40 cycles. Primer sequences: (i) GAPDH Forward (5'- CTA CAC TGA GGA CC AGG TTG TCT -3') Reverse (5'- TTG TCA TAC CAG GAA ATG AGC TT -3'), (ii) p75 Forward (5'- GCA TTG TGG TAG GCC AGA CC -3') Reverse (5'- CCT GAA AGT CAC TCC ATC CC -3') (iii) Sox9 Forward (5'- AGC TCA CCA GAC CCT GAG AA -3') Reverse (5'- TCC CAG CAA TCG TTA CCT TC -3'), (iv) Osterix Forward (5'- CTG CTT GAG GAA GAA GCT C -3') Reverse (5'- TTC TTT GTG CCT CCT TTC C -3'), (v) Osteocalcin Forward (5'- CTG ACA AAG CCT TCA TGT CCA A -3') Reverse (5'- GCG CCG GAG TCT GTT CAC TA -3'), (vi) Bone Sialoprotein Forward (5'CAG GGA GGC AGT GAC TCT TC -3') Reverse (5'- AGT GTG GAA AGT GTG GCG TT -3'), (vii) Alkaline phosphatase Forward (5'- AGT TAC TGG CGA CAG CAA GC -3') Reverse (5'- GAG TGG TGT TGC ATC GCG -3'), (viii) Aggrecan Forward (5'- TGG CTT CTG GAG ACA GGA CT -3') Reverse (5'- TTC TGC TGT CTG GGT CTC CT -3'), (ix) 
GCC CAG TTC AGG TCT CT -3'). mRNA expressions were assayed in triplicate for each sample and normalized to GAPDH housekeeping gene expressions to calculate $\Delta C T$. The undifferentiated and unencapsulated neural crest cells were used as control samples to calculate the relative gene expression levels in fold change $\left(2^{\Delta \Delta C T}\right.$ value).

\subsection{Immunohistochemistry}

For each time point, samples were fixed in $4 \%$ paraformaldehyde solution for $20 \mathrm{~min}$, permeabilized with $0.1 \%$ TritonX-100 (Sigma) and blocked with $1 \%$ bovine serum albumin for $2 \mathrm{~h}$ at room temperature. Primary antibodies for Osterix (OSX) (Abcam), RUNX2 (Novus-Biologicals), Osteopontin (OPN) (GenWay Biotech), Osteocalcin (OCN) (EMD Millipore), bone sialoprotein (BSP) (Bioss) and Ki67 (Abcam) were used at the concentrations recommended by the manufacturers, and the hydrogel samples were incubated with the antibodies overnight at $4{ }^{\circ} \mathrm{C}$. AlexaFluor 488 and AlexaFluor 568 (Life Technologies) were used as secondary antibodies. Alexa Fluor 647 Phalloidin (Life Technologies) was used to visualize the actin cytoskeleton. The images were taken with Zeiss LSM 780 confocal microscope.

\subsection{Mineralization assay}

The mineralization matrixes produced by the osteo-differentiated CNCCs were detected and quantified with alkaline phosphatase staining kit (Sigma) and hydroxyapatite quantifying Osteoimage ${ }^{\mathrm{TM}}$ (Lonza) assay, according to manufacturers' instructions. The mineralization levels in the cell-encapsulated hydrogels were assessed weekly for three weeks. The secreted hydroxyapatite was quantified by the relative fluorescence intensities of the cell-encapsulated hydrogels using ImageJ software (NIH, Bethesda, Maryland, USA). 


\subsection{Image Quantification}

The unprocessed raw confocal z-stack files were analyzed with Fiji software (ImageJ, $\mathrm{NIH}$, Bethesda, Maryland, USA) with 3-D objects counter plugins. The total number of cells were counted using DAPI staining. For OSX, cells with a positive signal in the nucleus were counted while cells with the positive signal in the cytoplasm were counted for OCN and OPN. Signals above a threshold set by the software were counted as positive and were confirmed manually.

\subsection{Freeze-Fracture Cryo-Scanning Electron Microscopy}

Freeze-fracture cryo-SEM imaging was performed with a Zeiss Supra55VP FESEM. A fixed single hydrogel was placed on a sample stub and submerged into a slurry bath of liquid nitrogen. The frozen sample was transferred to a cryo-transfer chamber and was fractured in a vacuum with a sharp blade to expose the cross sections of the sample. The surfaces of the fractured samples were sputter coated with platinum and imaged using the SE2 detector at a voltage of $4 \mathrm{kV}$ while maintained at $-160^{\circ} \mathrm{C}$.

\subsection{Statistical analysis}

Statistical analyses were performed with GraphPad Prism, version 5 (GraphPad Software, Inc., San Diego). Results were analyzed using analysis of variance with Tukey's post hoc test for multiple comparisons and Student's two-tailed t test for single comparisons, with statistical significance set at $p<0.05$. Unless otherwise stated, the mean values represent three experiments with two or three channels per experiment, and the error bars represent the standard error of mean.

\section{Results}

3.1. Characterization of hydrogel and neural crest cells 
3-D cellular microenvironments were designed from highly biocompatible gelatin-based hydrogels, supporting cell viability and growth [25]. Gelatin was methacrylated and lyophilized for the generation of UV photo-crosslinkable hydrogels (Supplementary Figure 1). Mouse CNCCs were isolated from the frontonasal, maxillary and mandibular prominences of E10.5 mouse embryos and expanded in the monolayer culture prior to 3D differentiation (Figure 1). The CNCCs before encapsulation showed $63.21 \% \pm 20.28$ positivity for ectodermal marker p75 and $66.22 \% \pm 11.64$ positivity for mesodermal marker CD73 confirming the mesenchymal-neural phenotype as previously shown (Figure 2A) $[26,27]$. The effect of the encapsulation process on cell viability were assessed with Live-Dead staining (Figure 2B). More than $90 \%$ of encapsulated cells were alive after the formation of 3-D constructs. The encapsulation process or the hydrogel itself do not restrict or alter the proliferation capacity of neural crest cells as shown with Ki67 immunostaining (Figure 2C). The encapsulated cells were then differentiated towards the osteogenic lineage in the 3-D microenvironment to evaluate their potential for ectomesenchymal transformation and the natural state osteogenesis. We investigated the effect of the cell encapsulation density on cranial osteogenesis under four different cell concentrations ( $1 \times 10^{6}$ to $4 \times 10^{7}$ cells $/ \mathrm{ml}$ ). Cell densities above $10^{7}$ cells $/ \mathrm{ml}$ showed significant expressions of the osteogenic markers (Figure 2D - I), whereas the lower cell concentrations resulted with poor osteogenic morphology. All following experiments were performed at a cell density of $4 \times 10^{7} \mathrm{cell} / \mathrm{ml}$.

\subsection{Monitoring the osteogenic differentiation of cranial neural crest cells in 3-D platform}

3-D differentiated CNCCs were analyzed for chondrogenic and osteogenic gene expressions with qRT-PRC (Figure 3). The neural crest marker p75 only maintained a high level of expression in the cells cultured in the neural crest cell-specific media and its expression decreased by more than $70 \%$ after the first week of culture in the osteogenic 
media (Figure 3A). Since CNCCs have the potential to differentiate into chondrocytes, we also checked the expression level of Sox9, collagen type II, and aggrecan. Sox9 is required for chondrogenesis, as it induces the expressions of cartilage-specific extracellular matrix molecules, such as collagen type II, IX and XI and aggrecan [28-31]. The Sox9 expression was slightly increased up to the second week but eventually decreased below the original level, confirming the cells were not in the course of chondrogenic differentiation (Figure 3B) [32, 33]. The expression of collagen type // and aggrecan did not show differences in expression between the control and the osteogenic samples (Figure 3C-D). To evaluate the osteogenic differentiation, expression levels of four major bone specific genes, Osterix (Osx, also known as Sp7), Alkaline phosphatase $(A / p)$, Osteocalcin $(\mathrm{Ocn})$ and Bone sialoprotein $(B s p)$ were analyzed. All four genes showed dramatic increase in their expression when cultured in the osteogenic differentiation media, especially by the third week of incubation, where markers indicated the presence of mixed population of osteoblasts and osteocytes (Figure 3E-H)

The expressions of bone-specific markers of 3-D encapsulated CNCC were also evaluated at the protein level for three weeks with immunocytochemistry (Figure 4). The differentiation of CNCCs to bone cells was demonstrated by the accumulation of OSX (Figure 4A and D), OPN (Figure 4B and E) and OCN proteins (Figure 4C and F). The expression of the early osteogenic marker RUNX2 was higher in cells cultured in the osteogenic media compared to the control cells at the initial differentiation phase, and RUNX2 expression later gradually decreased as the bone tissue started to form (Figure 4G and Supplementary Figure 2). On the other hand, more than $30 \%$ of encapsulated cells cultured in the osteogenic media showed OSX expression in the nucleus after three weeks of incubation (Figure 4D). The OPN and OCN protein depositions could be detected in the cytoplasm and the extracellular matrix of osteogenic cells cultured for 3 weeks in osteogenic media (Figure 4E and F), as in the late development phase of the in 
vivo bone tissue, confirming the successful osteogenic differentiation of the CNCCs using the 3-D hydrogel platform. We detected BSP depositions in the peripheral parts of the 3-D hydrogels starting from week 1, which gradually expanded its expression towards the core parts of the hydrogel during the 3-week osteogenic differentiation period (Supplementary Figure 2). By week 3, BSP accumulation could be observed around the lacunae of the osteogenic cells.

\subsection{Osteogenic mineralization and the morphological analysis of the neo-tissues}

ALP expression starts from the early osteogenic differentiation steps and continues through the maturation of the newly formed bone tissues. The engineered 3-D cellencapsulated hydrogels were analyzed for mineralization by examining the ALP activity in the cells differentiated over the 3-week culture period (Figure 5A). The cells cultured in the osteogenic condition inside the hydrogels exhibited high level of ALP activity after two weeks, which increased 2.56 folds by week 3 . The undifferentiated cells in the control hydrogels did not show any ALP activity (Figure 5A). We evaluated the functionality of osteo-differentiated CNCCs also with mineralization of hydrogels and the secretion of hydroxyapatite. The newly secreted hydroxyapatite in the 3-D cellencapsulated hydrogels was quantified with Osteoimage ${ }^{\circledR}$ Mineralization assay (Figure 5B). The secretion of hydroxyapatite crystals was detected in the osteogenic hydrogels starting from week 1 , which gradually increased with further osteogenic differentiation and maturation by week 3 . The undifferentiated control hydrogels did not show any fluorescent signals indicative of hydroxyapatite secretion.

Importantly, osteo-differentiated CNCC encapsulated hydrogels showed formation of in vivo-grade osteo lacuna structures, characteristic of the living bone tissue with embedded mature osteocytes (Figure 6, Supplementary Figure 3). Within the 3-D constructs osteo-differentiated cells also build intercellular connections providing cell-cell 
interactions (Figure 6C, Supplementary Video1 and Video 2). Morphological analysis of the osteo-differentiated hydrogels with FESEM revealed round, osteocyte-shaped cells located in the lacunae, and also mature osteoblasts throughout the hydrogel (Figure 7A C). Moreover, we observed differentiated bone cells with a large number of long projections called filopodia extending from the cell body, which is another key morphological characteristic of the mature osteocytes (Figure 7A, red colored) [34].

\section{Discussion}

Along with many other important roles in the embryonic development, neural crest cells are essential for the proper development of the vertebrate head. The human skull is composed of more than twenty intricately shaped and interconnected bones, which the majority is originated from the neural crest cells. Thus, deeper knowledge of the cranial neural crest cell behavior, and eventually how they properly differentiate to bone, is essential for understanding both the normal head development and the myriad of craniofacial abnormal conditions present in up to one-third of all congenital birth defects in humans [35]. The vertebrate cranium is also an important and unique structure from the evolutionary perspective as it represents one of the innovations that defines the vertebrate lineage, and vertebrate heads display a variety of adaptive and species-specific characteristics [36]. 3-D differentiation culture systems support efficient tissue formation and allow the manipulation of the cells in their native-like microenvironments [23]. Notably, our work demonstrates the generation of 3-D cellular niches for the efficient differentiation of CNCCs while maintaining their viability and proliferation capacity. To achieve a 3-D in vitro differentiation platform that recapitulates the in vivo osteogenic differentiation of CNCCs, we monitored and analyzed the developmental phases of cranial bone in our system. In the course of embryogenesis, the neural crest cells have a unique status as these cells of neuroectodermal origin can 
give rise to the specialized cell types that are typically derived from the mesodermal germ layer, such as connective tissue, cartilage and bones [26]. In this study, after monolayer amplification we encapsulated post-migratory CNCCs expressing both neural and mesenchymal markers, p75 and CD73 (Figure 2A). Clonal study performed on neural crest cells demonstrated that those cells are mostly formed as heterogeneous populations consisting of multiple progenitor cell types (e.g., melanocytes, glia, neuron, and myofibroblast) [37]. To reach the high CNCC differentiation yield, we investigated the effects of hydrogel properties and differential cell density in micro-tissue formation. Substrate stiffness and focal polarization of progenitor cells in conventional 2-D culture conditions dramatically affect the fate and physiology of cells [22, 38]. The 3-D platform that we generated allows tuning of both hydrogel stiffness and cell encapsulation density. According to our findings, high cell-to-cell contact dramatically assists the osteogenic differentiation in the microscale hydrogels. The highest level of cell-to-cell contacts and the native-like osteogenic differentiation in both morphological and molecular aspects were achieved in our 3-D culture system with the cell density of $4 \times 10^{7} \mathrm{cell} / \mathrm{ml}$.

We systematically investigated the intramembranous osteogenic differentiation progress of CNCCs in our 3-D platform by analyzing the expression patterns of multiple neural crest and skeletogenic markers, including p75, Sox9, Col II, aggrecan, Runx2, Osx, Alp, Ocn, Opn and Bsp (Figure 3 and 4). Cells encapsulated in hydrogels cultured in osteogenic media lost the expression of $p 75$ and even in early phase (week 1) are committed to osteogenic lineage. On the other hand, the ectodermal phenotype of CNCCs in control condition resumed up to week 2 with elevaded expression of $p 75$. Intramembranous ossification differs from the endochondral ossification, as it lacks the formation of hypertrophic cartilage template prior to generating mineralized calcified bone [39]. Sox9 is a well-known marker for chondrogenic cells in the process of endochondral ossification, but it also plays an important role in the differentiation of 
CNCCs to early bone progenitor cells during intramembranous ossification [19]. Accordingly, we detected the elevated levels of Sox9 expression during the early phase of the osteogenic differentiation, which then decreased below the initial level at the later stages of the culture process (Figure 3B). We also evaluated the chondrogenesis for potential endrochondral ossification with regulation of $\mathrm{Col} / /$ and aggrecan expression. After week 2, Col I/ expression slightly decreased in osteogenic conditions and aggrecan expression did not alter significantly for both study groups. These findings indicate there is no chondrogenic tissue formation and the osteogenic differentiation is through the intramembraneous course. Early phases of the osteogenesis process are directly regulated by Runx2, an early marker and regulator of many other osteoblast markers, such as Bsp, Ocn and Alp [19]. In our 3-D differentiation system, RUNX2 was expressed during the first week of osteogenesis and its expression decreased significantly at the later stages of bone tissue culture (Figure 4G). These later stage osteogenically committed cells no longer require RUNX2 for further ossification [40]. All of the later stage osteogenic markers (e.g., Osx, Ocn, Opn, BSP) suggest that skeletal development process was successfully recapitulated and led to the formation of mature osteoblasts and osteocytes. During the second and third week of 3-D osteo-differentiatiation CNCCs demonstrate the expression of i) OSX, a bone-specific transcription factor that is specifically expressed in osteoblasts of all skeletal elements [41], ii) OCN, an osteoblastderived hormone expressed only in fully differentiated osteoblasts and osteocytes, iii) OPN, a late osteogenic protein that regulates the biomineralization and boneremodelling $[42,43]$ and iv) BSP, a non-collagenous protein that is a key component of the bone extracellular matrix [44, 45](Figure 3E, F, Figure 4B, C, E, F, G and Supplementary Figure 2). The formation of mature bone tissue is further characterized by the mineralization in terms of hydroxyapatite deposition in the newly formed extracellular matrix [46]. We demonstrate the ability of newly differentiated osteogenic cells to 
mineralize the hydrogels with hydroxyapatite from second week. Similarly, high expression of alkaline phosphatase (ALP), which is highly involved in the mineralization of neo-tissues [47] supports the osteogenesis and in vitro tissue maturation (Figure 5). Another decisive method to evaluate the formation of bone tissue and the emergence of the differentiated osteoblasts and osteocytes is to analyze their morphologies compared to the native mature bone tissues with osteoblasts and osteocytes embedded. As early as one week after the initiation of the 3-D osteogenic differentiation process, the cell morphology of the neural crest cells began to change to reflect their differentiation into osteoblasts (Figure 6, Figure 7, Supplementary Video1 and Video 2). The appearance of the lacunae cavities containing mature osteoblasts and osteocytes within the hydrogel is strikingly similar to those seen in the natural bone tissue [48]. Such cell morphology changes during the course of osteogenesis can be observed through the freezefractured FESEM imaging. While the newly formed, larger osteoblast cells were tightly embedded in the hydrogel matrix (Figure 7B), their morphology changed to the small and round osteocyte-looking cells that reside in the spacious lacunae at later stages, and send multiple filopodia extensions through the surrounding matrix and the neighboring bone cells (Figure 7A). Filopodia penetrate the bone tissue through canallike structures called bone canaliculi and are believed to sense biomechanical stress and communicate with other bone cells within the same tissue [34]. These cellular projections also observed in our mature 3-D bone tissues extended beyond the immediate lacunae boundaries and continued through the surrounding matrix towards other neighboring bone cells (Figure 7A, white arrowheads). These cell-cell interactions were clearly detected in the cell-encapsulated 3-D hydrogels, starting from the second week of osteogenic differentiation.

In summary, we demonstrate a 3-D differentiation platform that enables to 
recapitulate an important developmental process in vitro. The platform we generated successfully supports the intramembranous ossification of cranial bone tissue using primary CNCCs. Thus, it is an essential tool added for studying the normal and abnormal osteogenic development in the model and non-model vertebrates. This platform has the potential to be used in a broader context, especially when combined with other bioengineering technologies $[6,8,49,50]$. This platform can be easily scaled up by assembly technologies, and be utilized in bottom-up tissue engineering, enabling to generate larger, multi-hydrogel constructs needed to understand the morphogenesis of specific skeletal structures. Neural crest cells differentiated from induced pluripotent stem cells (iPSCs) that are derived from human patients with craniofacial diseases could be used to monitor disease-specific phenotypes during osteogenic differentiation and eventually bone and other skeletal tissue formations for medical and pharmaceutical research. The adaptive and flexible nature of the platform will also allow it to be used to investigate the evolution of the diverse craniofacial bone shapes by including morphogenic factors in the specific regions of the assembled multi-hydrogels to recapitulate and manipulate the morphogenic processes in vitro.

\section{Acknowledgements}

UD acknowledges that this material is based in part upon work supported by the NSF CAREER Award Number 1150733, NIH R01EB015776, NIH R15HL115556 and NIH R21HL112114. AA acknowledges NSF 1257122 and Templeton Foundation RFP-12-01. Dr. $U$ Demirci is a founder of, and has an equity interest in: (i) DxNow Inc., a company that is developing microfluidic and imaging technologies for point-of-care diagnostic solutions, and (ii) Koek Biotech, a company that is developing microfluidic IVF technologies for clinical solutions. U.D.'s interests were viewed and managed in accordance with the conflict of interest policies. The other authors indicated no potential 
conflicts of interest. Authors thank to Yesim Bagatur (M.S.) and Zeynep Esencan (M.S.)

for their technical assistance.

\section{References}

1. Davenport, R.J., What controls organ regeneration. Science, 2005. 309(5731): p. 84-84.

2. Perez-Castillejos, R., Replication of the 3D architecture of tissues. Materials Today, 2010. 13(1-2): p. 32-41.

3. Song, Y.S., et al., Engineered 3D tissue models for cell-laden microfluidic channels. Anal Bioanal Chem, 2009. 395(1): p. 185-93.

4. $\mathrm{Xu}, \mathrm{F}$., et al., A three-dimensional in vitro ovarian cancer coculture model using a high-throughput cell patterning platform. Biotechnology Journal, 2011. 6(2): p. 204-212.

5. Guven, S., et al., Engineering of large osteogenic grafts with rapid engraftment capacity using mesenchymal and endothelial progenitors from human adipose tissue. Biomaterials, 2011. 32(25): p. 5801-9.

6. Guven, S., et al., Multiscale assembly for tissue engineering and regenerative medicine. Trends Biotechnol, 2015. 33(5): p. 269-79.

7. Bichsel, C.A., et al., Diagnostic microchip to assay 3D colony-growth potential of captured circulating tumor cells. Lab Chip, 2012. 12(13): p. 2313-6.

8. Tasoglu, S., et al., Untethered micro-robotic coding of three-dimensional material composition. Nat Commun, 2014. 5: p. 3124.

9. Tasoglu, S., et al., Guided and magnetic self-assembly of tunable magnetoceptive gels. Nat Commun, 2014. 5: p. 4702.

10. Hall, B.K., The neural crest as a fourth germ layer and vertebrates as quadroblastic not triploblastic. Evol Dev, 2000. 2(1): p. 3-5.

11. Hall, B.K., The neural crest and neural crest cells: discovery and significance for theories of embryonic organization. J Biosci, 2008. 33(5): p. 781-93.

12. Le Douarin, N. and C. Kalcheim, The neural crest. 2nd ed. Developmental and cell biology series 36. 1999, Cambridge, UK ; New York, NY, USA: Cambridge University Press. xxiii, 445 p.

13. Couly, G.F., P.M. Coltey, and N.M. Le Douarin, The triple origin of skull in higher vertebrates: a study in quail-chick chimeras. Development, 1993. 117(2): p. 40929.

14. Noden, D.M., The role of the neural crest in patterning of avian cranial skeletal, connective, and muscle tissues. Dev Biol, 1983. 96(1): p. 144-65.

15. Noden, D.M., Cell movements and control of patterned tissue assembly during craniofacial development. J Craniofac Genet Dev Biol, 1991. 11(4): p. 192-213.

16. Hall, B.K. and T. Miyake, The membranous skeleton: the role of cell condensations in vertebrate skeletogenesis. Anat Embryol (Berl), 1992. 186(2): p. 107-24.

17. Jiang, X., et al., Tissue origins and interactions in the mammalian skull vault. Dev Biol, 2002. 241(1): p. 106-16.

18. Helms, J.A., D. Cordero, and M.D. Tapadia, New insights into craniofacial morphogenesis. Development, 2005. 132(5): p. 851-61.

19. Abzhanov, A., et al., Regulation of skeletogenic differentiation in cranial dermal bone. Development, 2007. 134(17): p. 3133-44. 
20. Noden, D.M. and R.A. Schneider, Neural crest cells and the community of plan for craniofacial development: historical debates and current perspectives. Adv Exp Med Biol, 2006. 589: p. 1-23.

21. Pineda, E.T., R.M. Nerem, and T. Ahsan, Differentiation patterns of embryonic stem cells in two- versus three-dimensional culture. Cells Tissues Organs, 2013. 197(5): p. 399-410.

22. Lutolf, M.P., P.M. Gilbert, and H.M. Blau, Designing materials to direct stem-cell fate. Nature, 2009. 462(7272): p. 433-41.

23. Ravi, M., et al., 3D cell culture systems: advantages and applications. J Cell Physiol, 2015. 230(1): p. 16-26.

24. Etchevers, H., Primary culture of chick, mouse or human neural crest cells. Nat Protoc, 2011. 6(10): p. 1568-77.

25. Gurkan, U.A., et al., Simple Precision Creation of Digitally Specified, Spatially Heterogeneous, Engineered Tissue Architectures. Advanced Materials, 2013. 25(8): p. 1192-1198.

26. Le Douarin, N.M., G.W. Calloni, and E. Dupin, The stem cells of the neural crest. Cell Cycle, 2008. 7(8): p. 1013-9.

27. von Levetzow, C., et al., Modeling initiation of Ewing sarcoma in human neural crest cells. PLoS One, 2011. 6(4): p. e19305.

28. Lefebvre, V., et al., SOX9 is a potent activator of the chondrocyte-specific enhancer of the pro alpha1(II) collagen gene. Mol Cell Biol, 1997. 17(4): p. 233646.

29. Bi, W., et al., Sox9 is required for cartilage formation. Nat Genet, 1999. 22(1): p. 85-9.

30. Healy, C., D. Uwanogho, and P.T. Sharpe, Regulation and role of Sox9 in cartilage formation. Dev Dyn, 1999. 215(1): p. 69-78.

31. Mori-Akiyama, Y., et al., Sox9 is required for determination of the chondrogenic cell lineage in the cranial neural crest. Proc Natl Acad Sci U S A, 2003. 100(16): p. 9360-5.

32. $\mathrm{Ng}$, L.J., et al., SOX9 binds DNA, activates transcription, and coexpresses with type I/ collagen during chondrogenesis in the mouse. Dev Biol, 1997. 183(1): p. 108-21.

33. Zhao, Q., et al., Parallel expression of Sox9 and Col2a1 in cells undergoing chondrogenesis. Dev Dyn, 1997. 209(4): p. 377-86.

34. Aarden, E.M., E.H. Burger, and P.J. Nijweide, Function of osteocytes in bone. J Cell Biochem, 1994. 55(3): p. 287-99.

35. Jones, N.C. and P.A. Trainor, The therapeutic potential of stem cells in the treatment of craniofacial abnormalities. Expert Opin Biol Ther, 2004. 4(5): p. 64557.

36. Gans, C. and R.G. Northcutt, Neural crest and the origin of vertebrates: a new head. Science, 1983. 220(4594): p. 268-73.

37. Calloni, G.W., N.M. Le Douarin, and E. Dupin, High frequency of cephalic neural crest cells shows coexistence of neurogenic, melanogenic, and osteogenic differentiation capacities. Proc Natl Acad Sci U S A, 2009. 106(22): p. 8947-52.

38. Bucaro, M.A., et al., Fine-tuning the degree of stem cell polarization and alignment on ordered arrays of high-aspect-ratio nanopillars. ACS Nano, 2012. 6(7): p. 6222-30.

39. Scotti, C., et al., Recapitulation of endochondral bone formation using human adult mesenchymal stem cells as a paradigm for developmental engineering. Proc Natl Acad Sci U S A, 2010. 107(16): p. 7251-6. 
40. Takarada, T., et al., An analysis of skeletal development in osteoblast-specific and chondrocyte-specific runt-related transcription factor-2 (Runx2) knockout mice. J Bone Miner Res, 2013. 28(10): p. 2064-9.

41. Nakashima, K., et al., The novel zinc finger-containing transcription factor osterix is required for osteoblast differentiation and bone formation. Cell, 2002. 108(1): $p$. 17-29.

42. Hunter, G.K., Role of osteopontin in modulation of hydroxyapatite formation. Calcif Tissue Int, 2013. 93(4): p. 348-54.

43. Choi, S.T., et al., Osteopontin might be involved in bone remodelling rather than in inflammation in ankylosing spondylitis. Rheumatology (Oxford), 2008. 47(12): p. 1775-9.

44. Hauschka, P.V., et al., Osteocalcin and matrix Gla protein: vitamin K-dependent proteins in bone. Physiol Rev, 1989. 69(3): p. 990-1047.

45. Fisher, L.W., et al., Matrix sialoprotein of developing bone. J Biol Chem, 1983. 258(20): p. $12723-7$.

46. Gkioni, K., et al., Mineralization of hydrogels for bone regeneration. Tissue Eng Part B Rev, 2010. 16(6): p. 577-85.

47. Mikuni-Takagaki, Y., et al., Matrix mineralization and the differentiation of osteocyte-like cells in culture. J Bone Miner Res, 1995. 10(2): p. 231-42.

48. Pazzaglia, U.E., et al., Osteoblast-osteocyte transformation. A SEM densitometric analysis of endosteal apposition in rabbit femur. J Anat, 2014. 224(2): p. 132-41.

49. Chen, P., et al., Biotunable Acoustic Node Assembly of Organoids. Adv Healthc Mater, 2015. 4(13): p. 1937-43.

50. Tasoglu, S., et al., Magnetic Levitational Assembly for Living Material Fabrication. Adv Healthc Mater, 2015. 4(10): p. 1469-76, 1422.

\section{Figure Legends:}

Figure 1. Recapitulation of the osteogenic differentiation of CNCCs with direct differentiation in the 3-D hydrogels in vitro. Neural crest cells were isolated from E10.5 mouse embryo and initially expanded in monolayer with neural crest-specific media and later on encapsulated in hydrogels forming 3-D in vitro culture. Osteogenic differentiation phases with specific gene expressions in 3-D microenvironments were monitored for three weeks.

Figure 2. The characterizations of the CNCCs prior and after hydrogel encapsulation. (A) FACS analysis of the CNCCs prior to the encapsulation in 3-D hydrogels. $63.21 \% \pm 20.28$ of cells were p75 positive indicating the neural crest cells phenotype (left), and $66.22 \% \pm 11.64$ were CD73 positive showing mesenchymal lineage characteristic (right). Corresponding IgG staining was used as a negative control (blue contour). (B) The Live-Dead staining of neural crest cells encapsulated in the 3-D hydrogel. Live cells were stained for green and dead cell are shown in red. (C) The proliferation capacity of neural crest cells was evaluated with Ki67 staining after 1 week of encapsulation in the 3-D hydrogels. Proliferating cells express Ki67 (red) in the nuclei, co-localizing with DAPI (blue). The cell cytoskeletons were shown with phalloidin staining (green). (D-I) Effects of cell encapsulation density on osteogenic differentiation of primary CNCCs. (D, E, F) Bright field images of the cell-encapsulated hydrogels generated using different cell densities. (G, H, I) The immunocytochemistry results for OSX (green) and cytoskeleton 
(phalloidin, magenta) in 3-week osteo-differentiated hydrogels showed that high cell density is more favorable for osteogenic differentiation. Scale bars $100 \mu \mathrm{m}$.

Figure 3. qRT-PCR analysis of the neural crest-specific gene $p 75$ and bone and cartilage-specific genes in cells cultured in control or osteogenic media for three weeks ( $\mathrm{n}=3$ for each sample. Error bar indicates standard error). (A) p75 was highly expressed in encapsulated cells cultured in control media. (B) The expression of cartilage-specific marker Sox9 did not change in both control and osteogenic media. (C) Col I/ expression slightly decreased after week 2. (D) Expression of chondrogenic marker aggrecan did not alter significantly during the differentiation. (E-H) Bone specific marker Osx, Alp, $O c n$, and Bsp were highly expressed in the cells cultured in osteogenic media especially at the third week of incubation. (One asterisks denote significance at $\mathrm{P}<0.05$, two asterisks denote significance at $P<0.01$, Student t-test).

Figure 4. The cell-encapsulated hydrogels cultured either in control media $(C M)(A-C)$ or osteogenic media (OM) (D-F) were stained for OSX, OPN and OCN after three weeks. (A, D) The nuclear localization of osteoblastic marker OSX (green) could be seen in the differentiated cells cultured in the osteogenic media. (B, C, E, F) Late osteogenic markers OPN (red) and OCN (green) were expressed in the cytoplasm and the extracellular matrix of osteo-differentiated cells ( $E$ and $F)$. (G) The expression of early osteogenic marker RUNX2 decreased during the course of osteogenic differentiation of CNCCs, whereas the expression of OPN and OSX increased ( $n=3$ for each sample. Error bar indicates standard error). All scale bars $50 \mu \mathrm{m}$.

Figure 5. The osteogenic mineralization in the control and osteo-differentiated cellencapsulated 3-D hydrogels were determined and quantified at weeks 1,2 and 3 with alkaline phosphatase (A), and hydroxyapatite (B) staining. The osteogenic differentiation of CNCCs showed hydroxyapatite secretion and mineralized the hydrogels gradually after week 1 (C). Scale bars $100 \mu \mathrm{m}$.

Figure 6. The hydrogel-encapsulated CNCCs change their morphology in the 3-D osteogenic differentiation platform. (A) Hydrogel-encapsulated CNCCs incubated in control media exhibited elongated morphology typical for mesenchymal cells. (B) Spherical morphology of osteo-differentiated cells in lacunae-like niches. Cytoskeletons were stained with phalloidin (magenta) and cell nuclei with DAPI (blue). (C) The confocal image of the hydrogel with encapsulated cells differentiated for two weeks in osteogenic media. The immunostaining results showed early osteogenic cells positive for RUNX2 (red) and osteocytes positive for OSX (green). The cytoskeletons stained with phalloidin (white) revealed filopodia projections and cell-to-cell connections (red arrowheads). Scale bars: $100 \mu \mathrm{m}$ in A and B, $10 \mu \mathrm{m}$ in C.

Figure 7. The representative freeze-fractured hydrogel FESEM images of the bioengineered neo-tissues, after three weeks of osteogenic differentiation. Cellencapsulated hydrogels revealed cells in different phases of osteogenic differentiations, recapitulating native-like microenvironment. (A) An osteocyte (red) in a lacuna with extended filopodia that could also be observed in the bone canaliculus-like structure 
(white arrowheads), connecting to the neighboring cells. (B) A focused image of a single osteoblast starting to remodel the surrounding hydrogel into a lacuna-like structure. (C) Representative image of the osteogenic niche in 3-D hydrogel microenvironment. Scale bars; $3 \mu \mathrm{m}$ in $\mathrm{A}$ and $\mathrm{B}, 10 \mu \mathrm{m}$ in $\mathrm{C}$. 


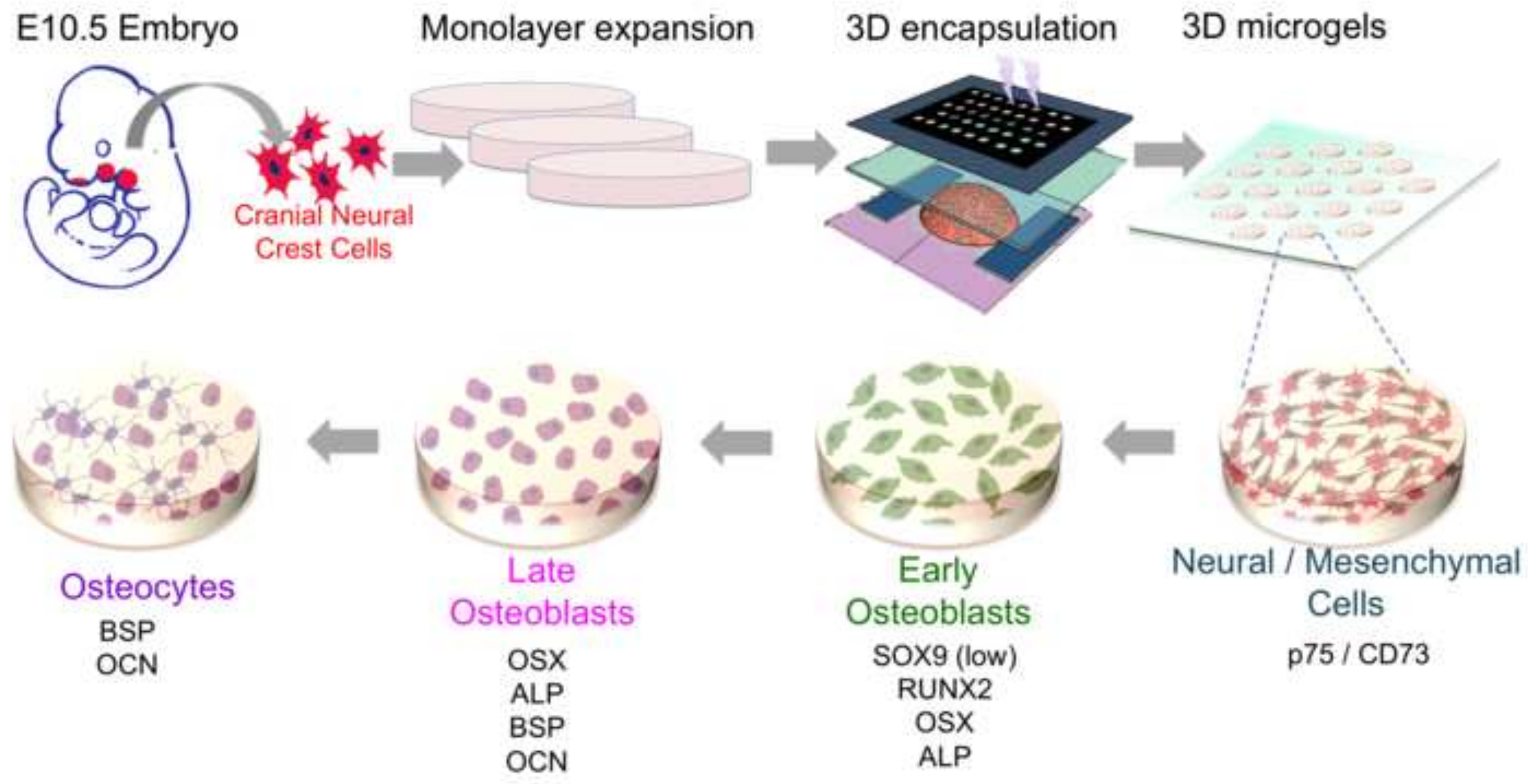



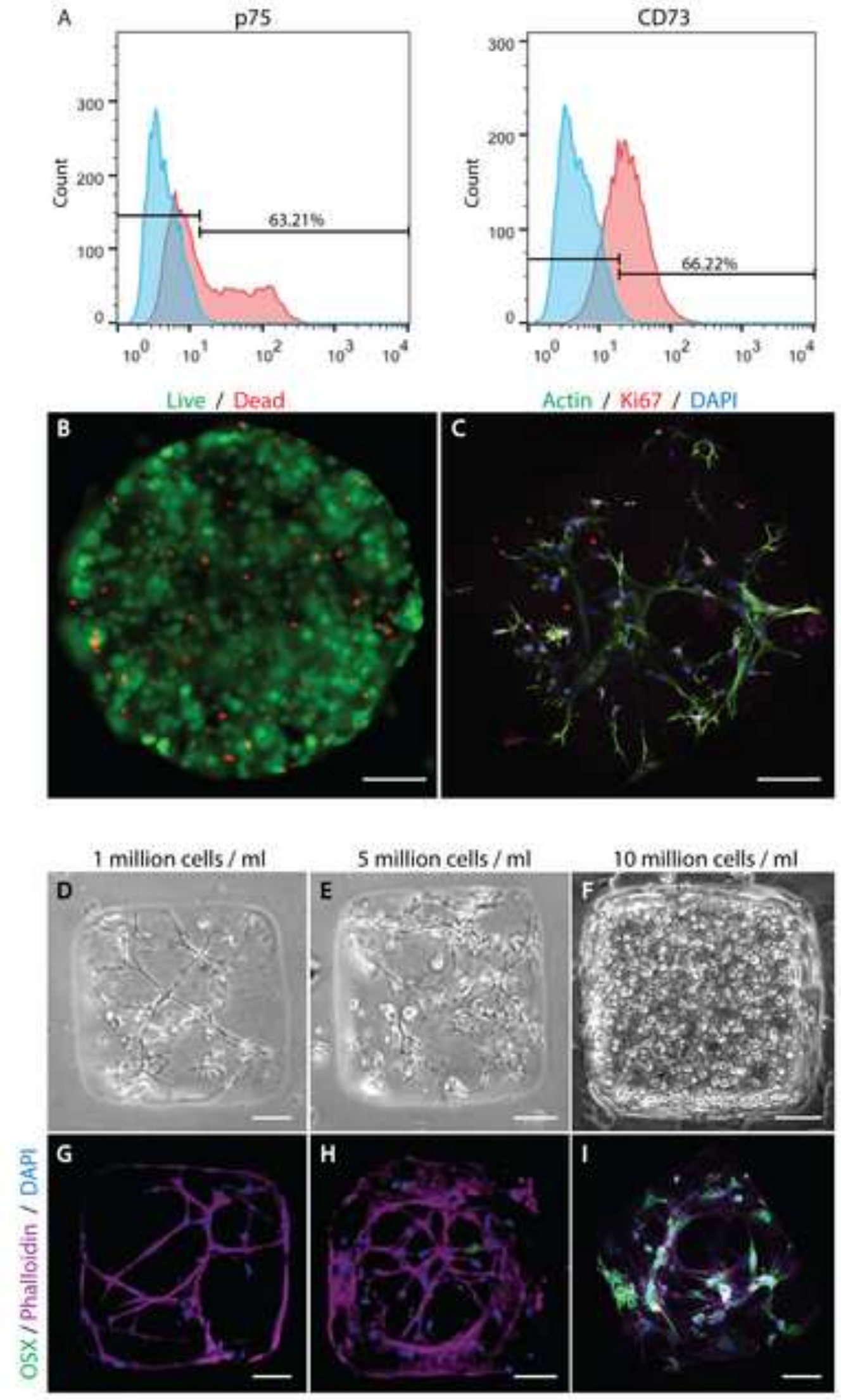

Figure 2 
Figure 3
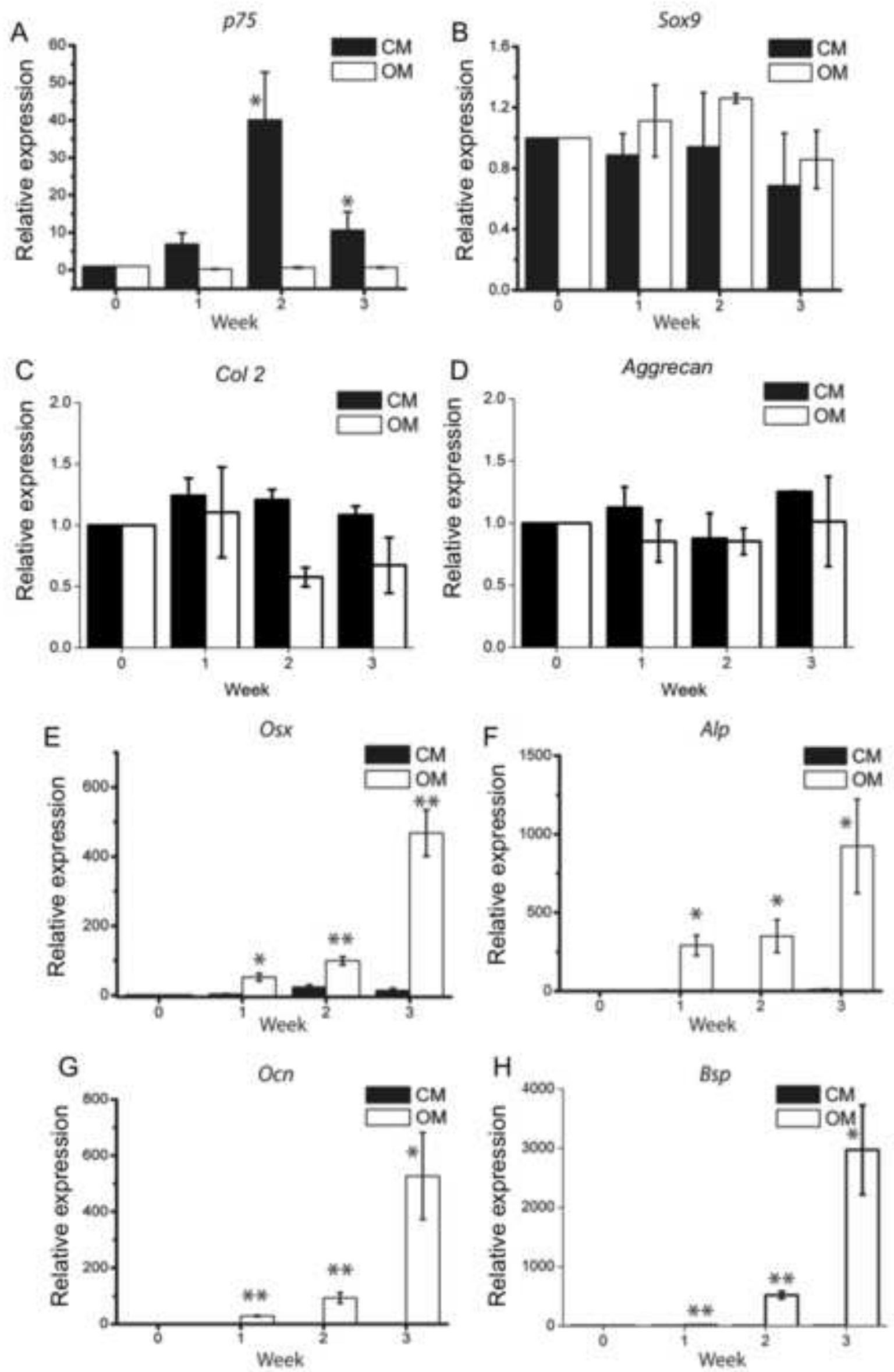

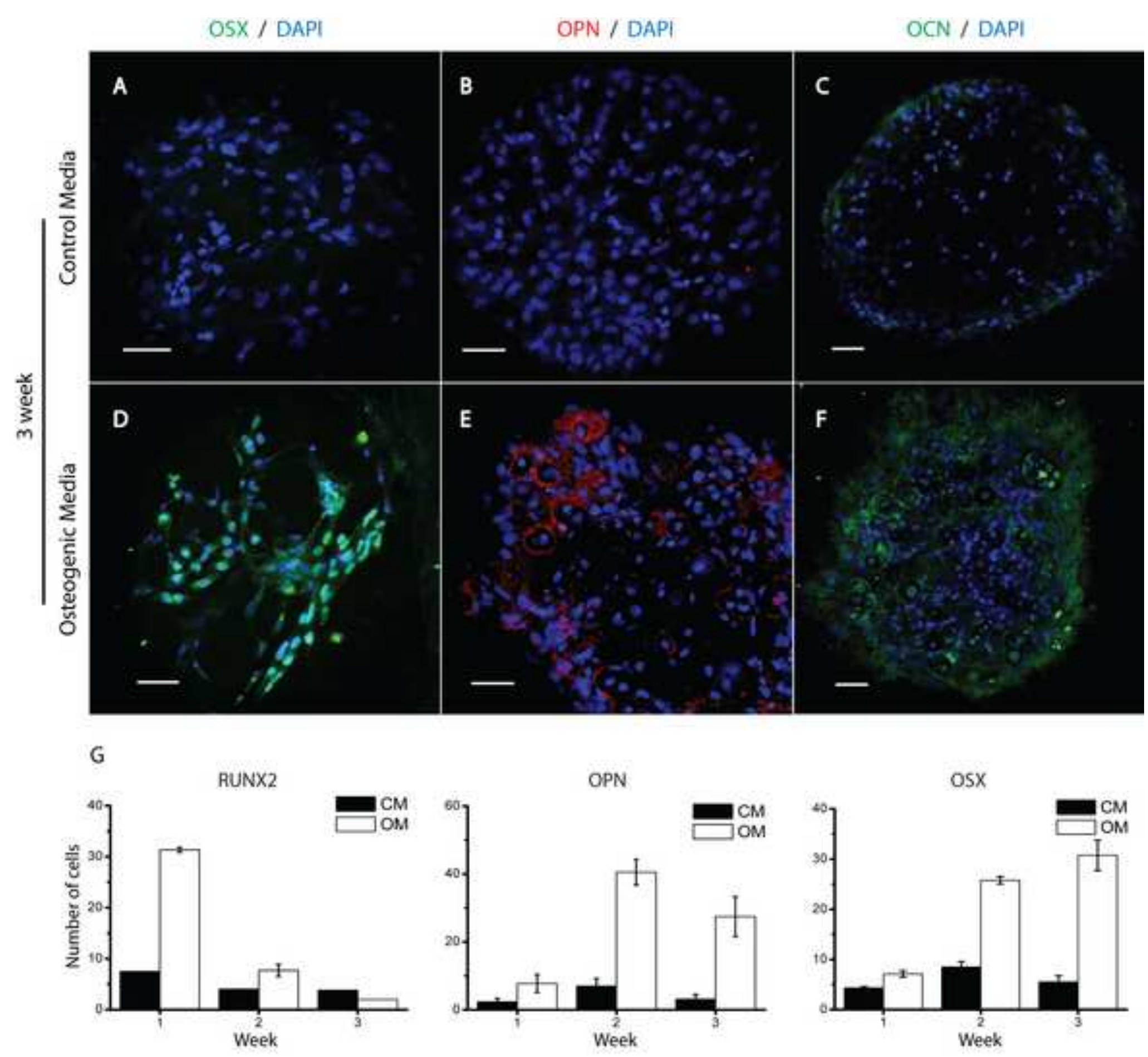


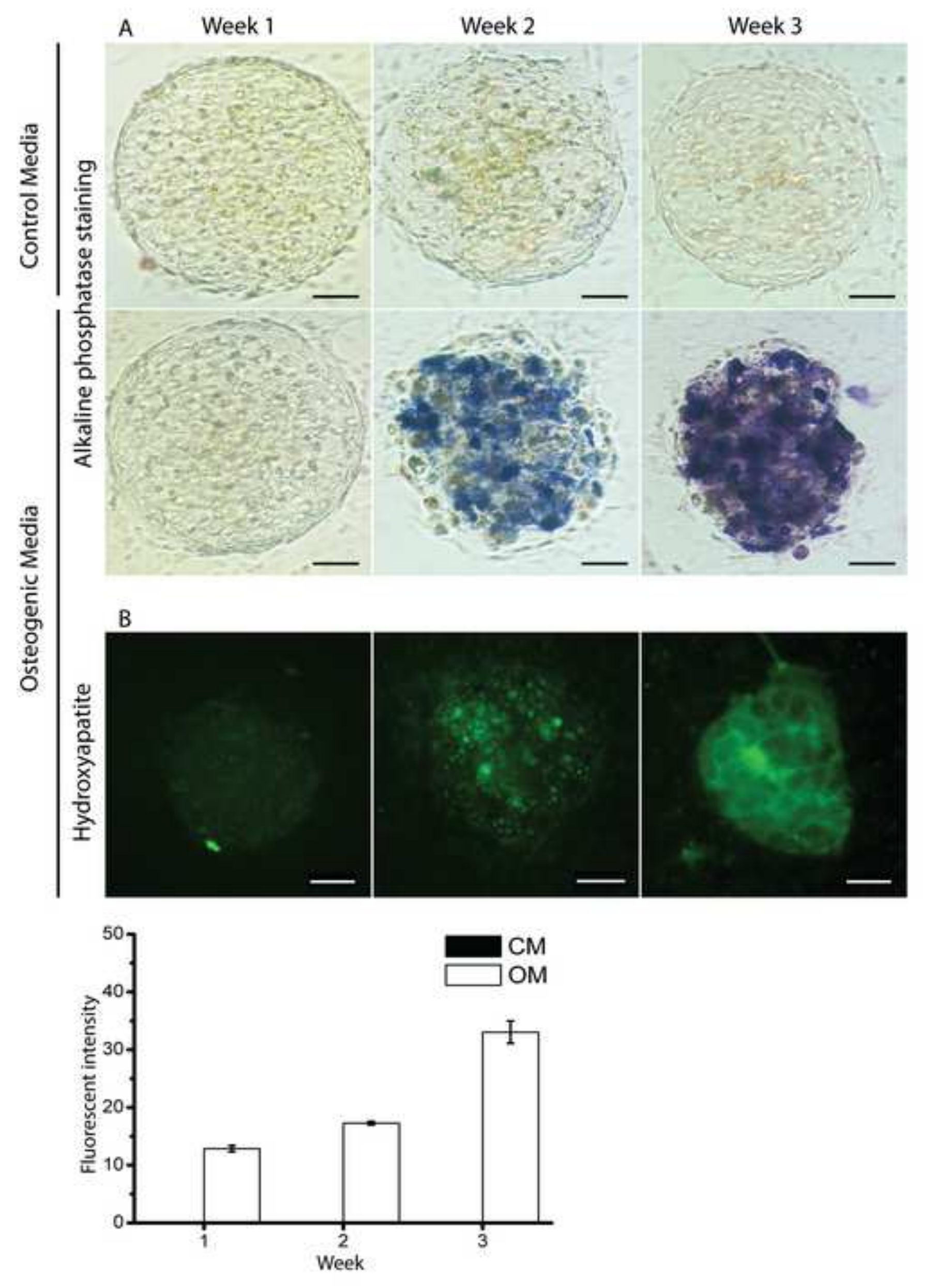

Figure 5

$\begin{array}{llll}\text { A } & \text { Week } 1 & \text { Week } 2 & \text { Week } 3\end{array}$
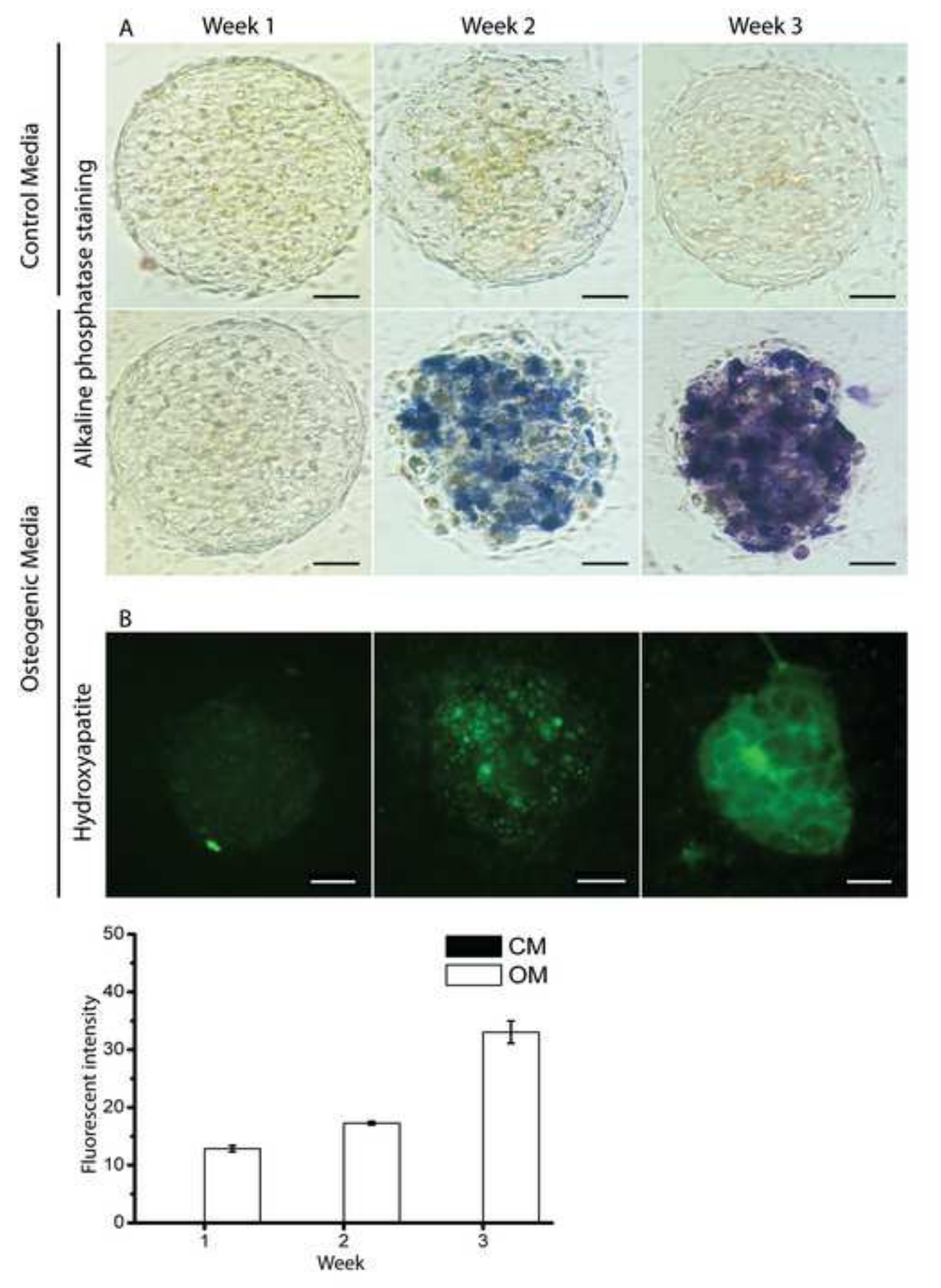


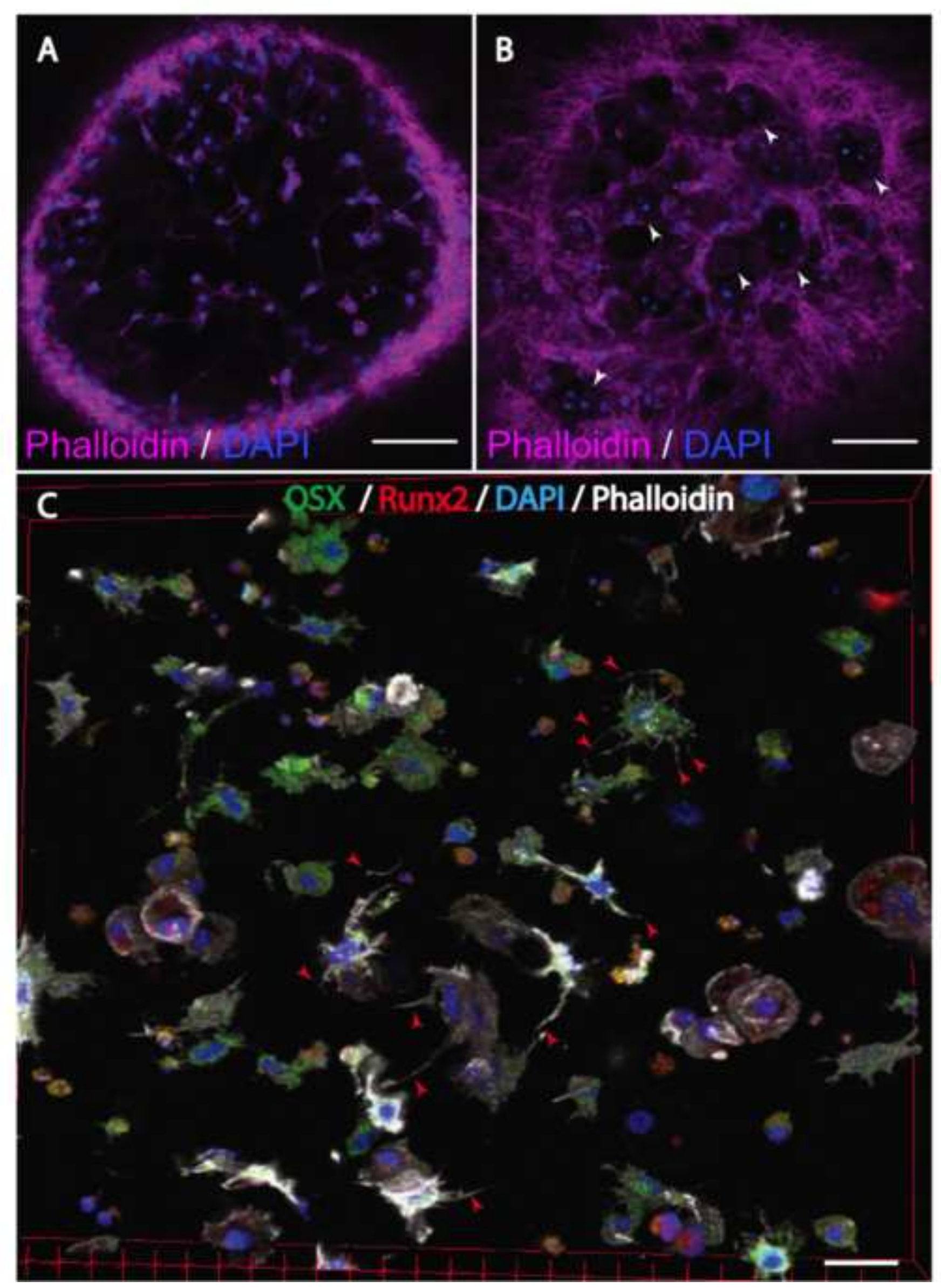

\section{Figure 6} .
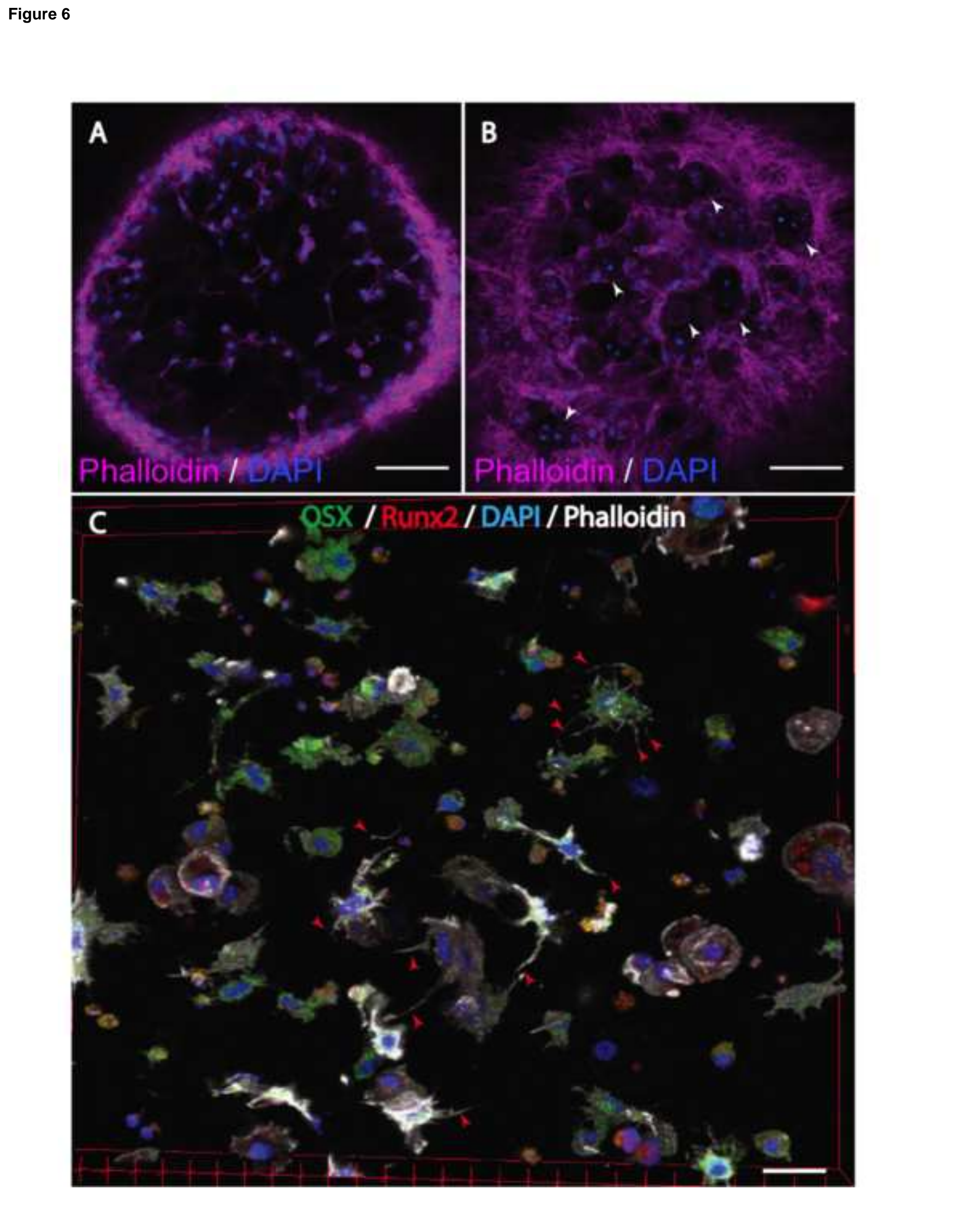


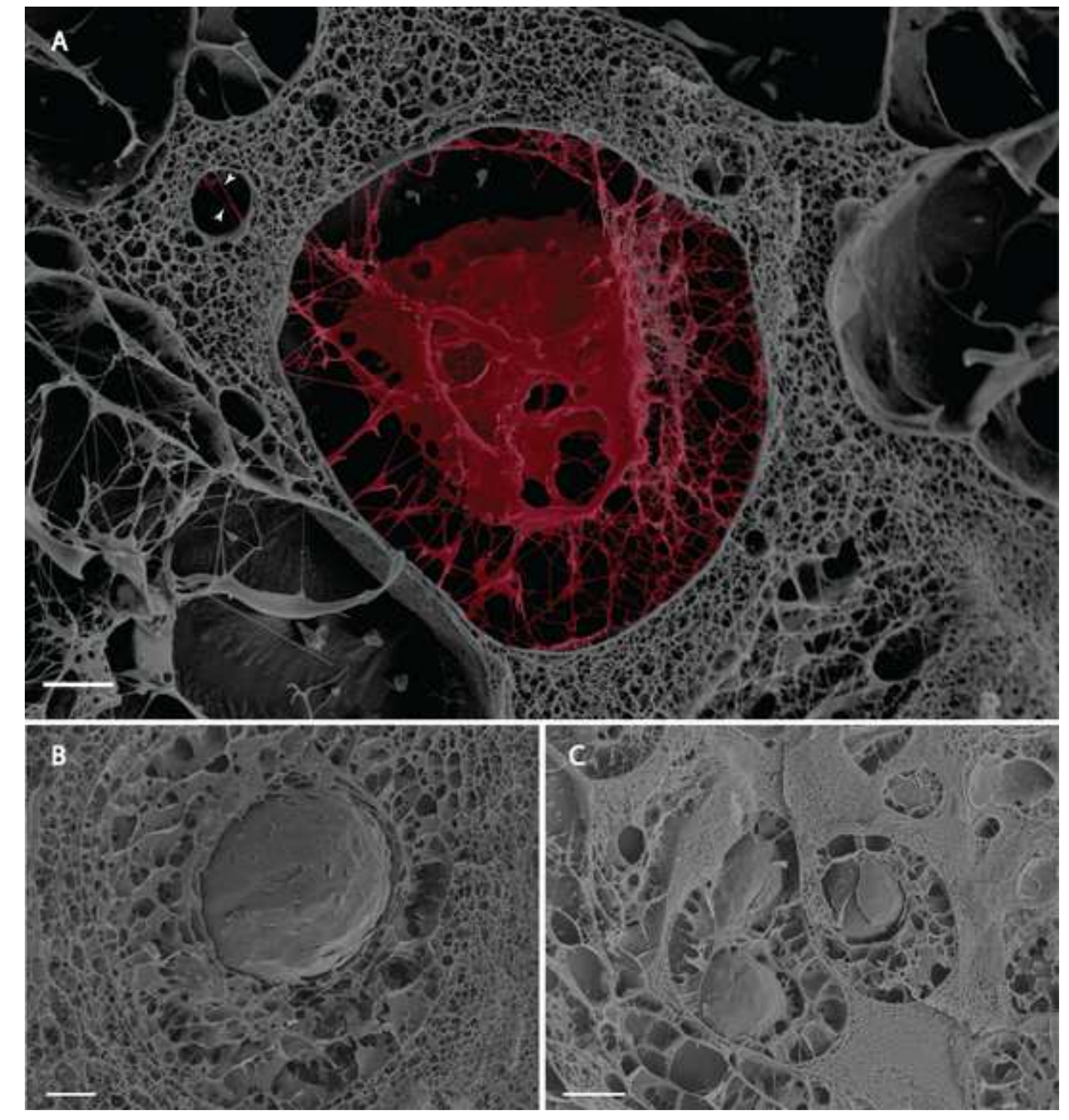

Figure

.

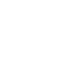

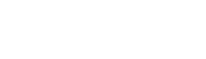

-

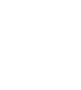
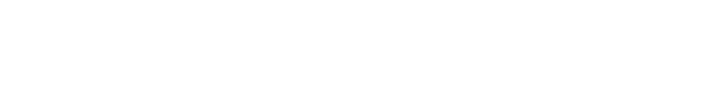

-
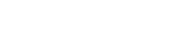

$-$

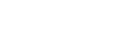

$-$

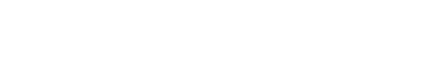

$-3$

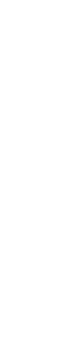

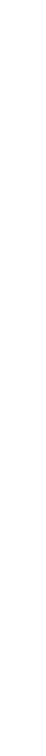




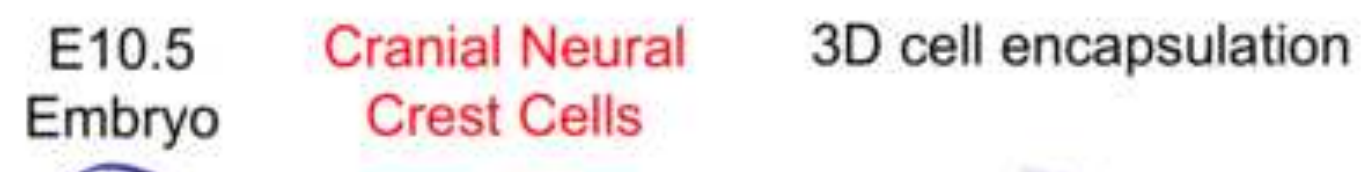

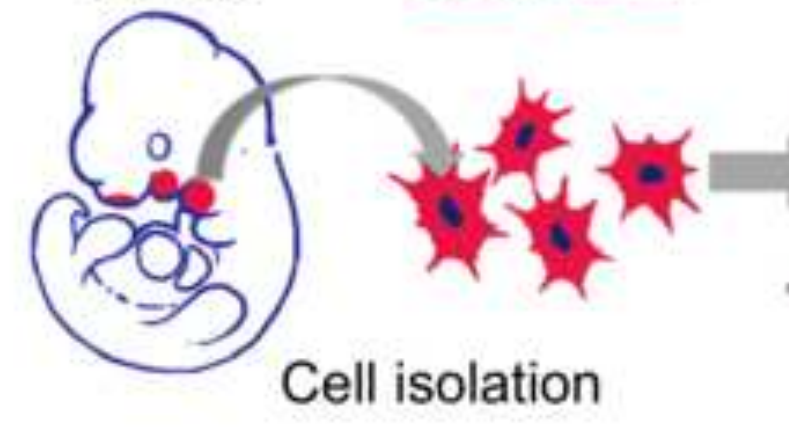

Cell isolation

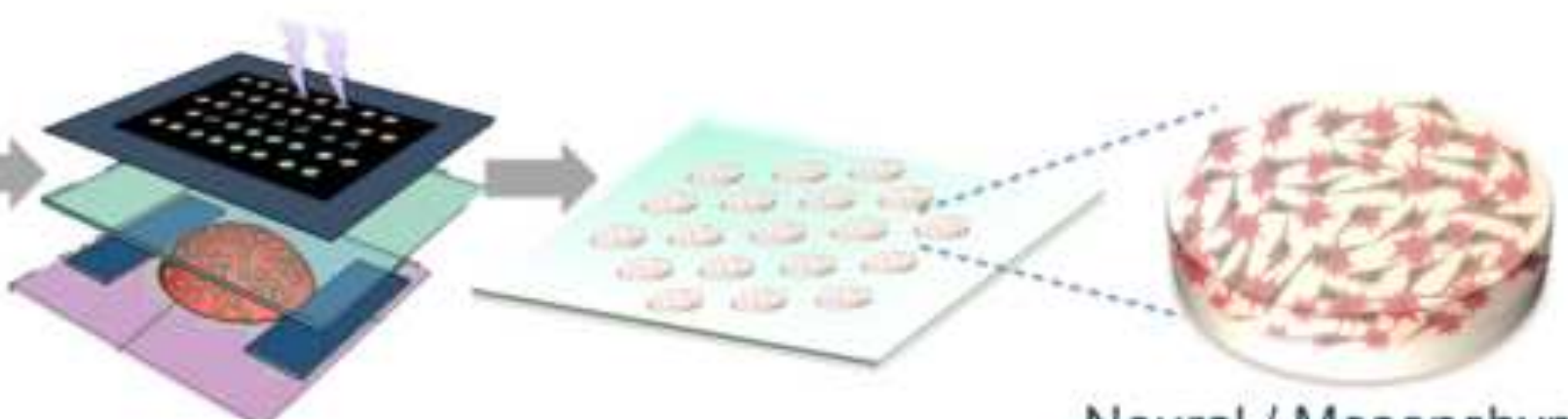

Neural / Mesenchymal Cells p75 / CD73

In vitro bone tissue
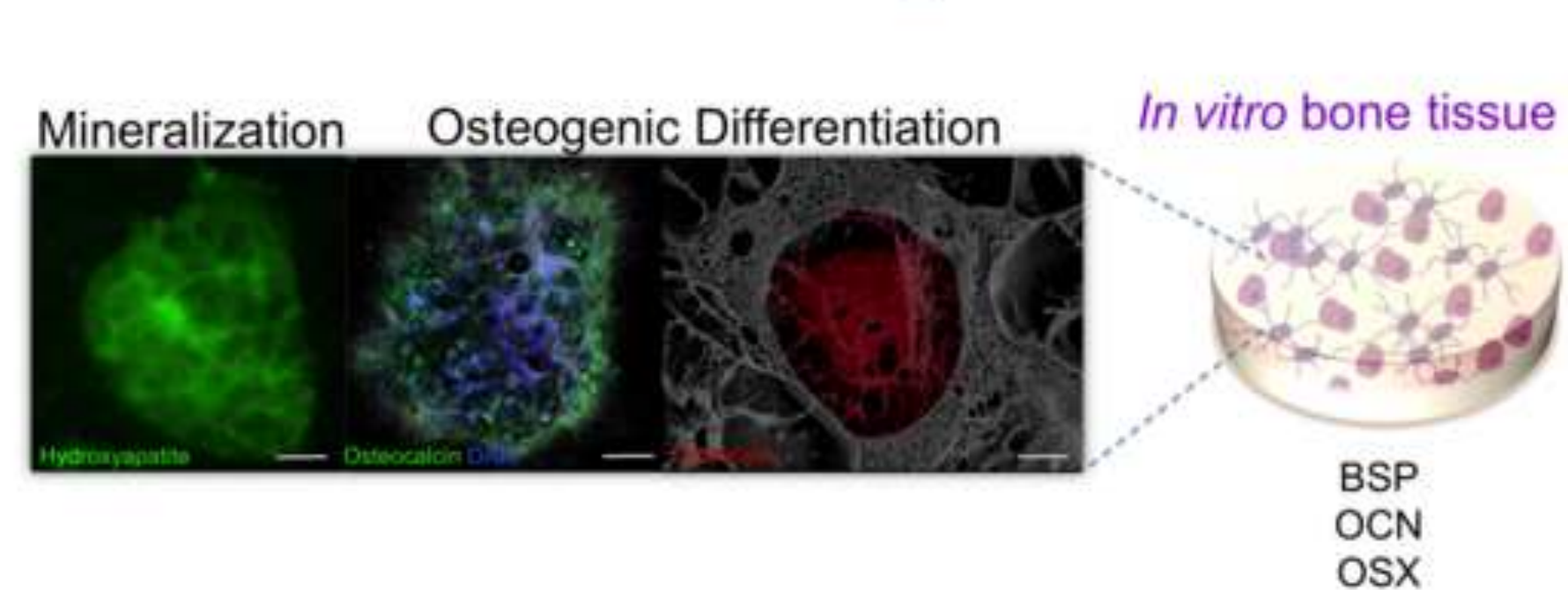

osx 